\title{
From Human Physical Interaction To Online Motion Adaptation Using Parameterized Dynamical Systems
}

\author{
Mahdi Khoramshahi*, Antoine Laurens, Thomas Triquet, and Aude Billard
}

\begin{abstract}
In this work, we present an adaptive motion planning approach for impedance-controlled robots to modify their tasks based on human physical interactions. We use a class of parameterized time-independent dynamical systems for motion generation where the modulation of such parameters allows for motion flexibility. To adapt to human interactions, we update the parameters of our dynamical system in order to reduce the tracking error (i.e., between the desired trajectory generated by the dynamical system and the real trajectory influenced by the human interaction). We provide analytical analysis and several simulations of our method. Finally, we investigate our approach through real world experiments with a 7-DOF KUKA LWR 4+ robot performing tasks such as polishing and pick-and-place.
\end{abstract}

\section{INTRODUCTION}

Robots are mainly here to assist us with tasks that are repetitive and burdensome such as polishing surfaces and pick-and-place. Over the past four decades, the problem of control and motion planning for such tasks has been studied rigorously for traditional industrial settings. However, recent advances in robotics aim to utilize robots in everyday settings, such as small factories and home applications. Therefore, having a human in proximity of robots who intends to modify the robotic behavior through physical interactions introduces new challenges; not only from a control perspective to ensure safety and passivity but also regarding motion planning to recognize the underlying intentions and react accordingly. Interacting with a human who might have different intentions/goals can be done in several fashions: using control panels, remote controllers or other extra interfaces. However, a seamless behavior can be achieved if the robot reacts in accordance with human interactions. Therefore, it seems desirable to have robots that are not only compliant in their interaction, but also adaptive to the intention of their users.

A myriad of robotic implementations made it clear that collaborative robots require a form of compliant control for safe and passive interaction with human users; see [1] as an example. An impedance controller ([2]), for instance, can follow a desired trajectory while being compliant to perturbations (made by the human user). The impedance parameters (e.g., inertia, damping, and stiffness) allow for adaptation methods to achieve various control objectives: to adapt to human forces [3], human compliant behavior [4], or human intended set-point [5]. While these approaches are very effective to locally and temporally adapt the human physical-interactions, they are limited in adaptation to human

All authors are with Learning Algorithm and Systems Laboratory, EPFL. *Corresponding author: mahdi.khoramshahi@epfl.ch

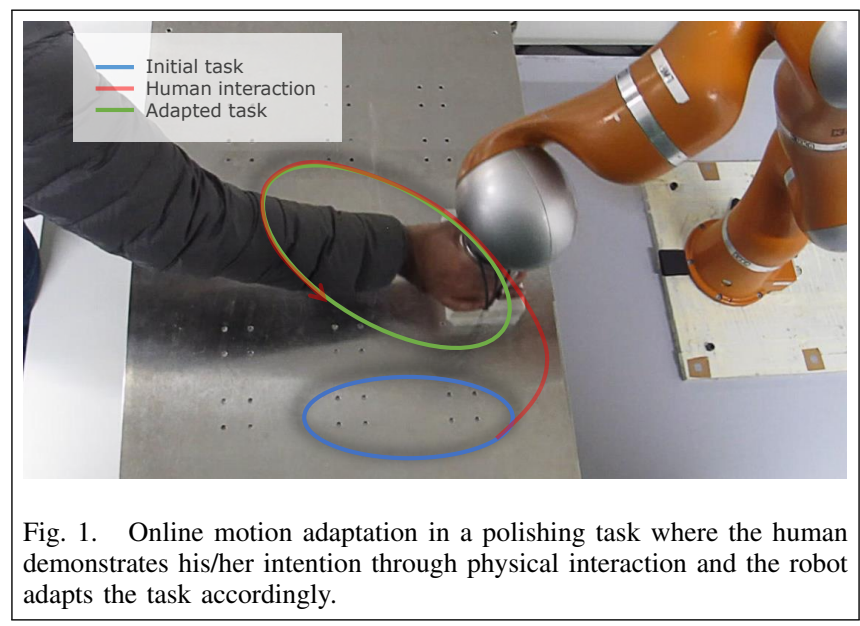

intention with regard to the underlying task. In this work, we address this issue by adapting the task itself (i.e., the motion planner rather than the impedance controller) in order to accommodate for demonstrated human-interactions; see Fig.1 where the underlying behavior of the task (i.e., the ellipsoid) is preserved and adapted accordingly.

Incremental learning approaches focus on the learning of a task as a whole through several interactions with the environment or the human user; see [6], [7], and [8]. Several techniques can be envisioned to accommodate the new experiences. For instance reinforcement learning can be used to learn the dynamics of physical interaction with human [9], or learn to stay in contact with a surface properly [10]. In [11] Optimal control is used to improve the trajectories for heavy load-carrying with a human. These methods can learn from small corrections made by the human during each interaction in order to achieve their goal; see [12]. These demonstration can take place through physical interaction with the robot; see [13], [14], [15], [16] for kinesthetic teaching. However, the fact that in most current approaches the learning and execution phases are disjoint and defined by the human supervisor limits reaching a seamless interaction. The interaction can be more effective if the robot learns proper motion regarding different human intentions and during the execution phase, the robot only adapts to the proper already-learned motion based on human interaction. [17] addressed this issue by learning statistical models that not only generates motions for a given task but also, inversely, recognize a task from a given motion. Others propose to recognize the intention of the human based on the interaction forces [18]. Furthermore, online switching across tasks based 


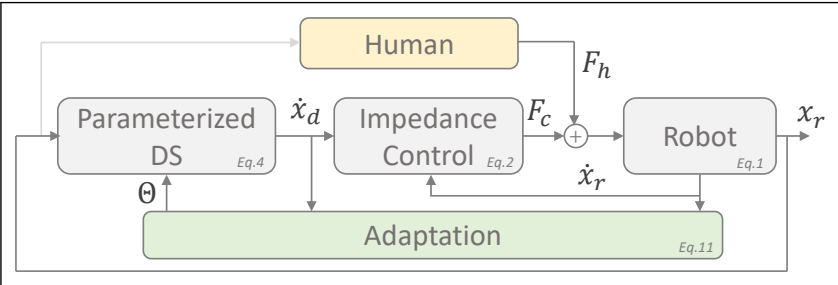

Fig. 2. Control loop of an adaptable dynamical system

on the human interaction has been proposed in [19], [20] which provides the robot with proactive behavior. In this work, we take a similar approach but rather than switching across different tasks, we adapt the current task based on the human interaction.

Simple robotic tasks, such as polishing and pick-andplace, can be improved by adapting to the intention of the human user. For example, adapting to the human desired forces can improve the quality of a polishing task [21], [22], [23]. This task can be improved further by adapting to the human desired patterns. In another example, adaptive behavior was provided for pick-and-place task where the target locations were determined using visual guidance [24] or natural language [25]. In this work, we propose adaptive motion planning for such tasks based on the physical interactions. To do so, we employ parameterized dynamical systems (DS) which show flexibility toward numerous possible human-intentions. For instance, in [26], [27] the parameters of a time-dependent DS are adapted to achieve a desired force during human-robot interaction. However, adaptive time-dependent DSs capture only the temporal aspect of the input signal (e.g., phase lags and offsets). In this work, to capture the spatial aspect of the human intention (e.g., where to polish or pick/place), we employ state-dependent dynamical systems [28]. We treat the human as a state-dependent reference model (with the intended parameters), we propose and analyze our adaptive mechanism in Section II. We put our method in practice and report on the results in Section III. Finally, in Section IV we discuss and conclude our work.

\section{METHOD}

In this section, we present our adaptive architecture which is illustrated in Fig. 2. In the following, we present the mathematical formulation for the control of the robot, motion generation, and motion adaptation.

\section{A. Robot Control}

Consider the rigid-body dynamics with $n$ degrees of freedom described in the $m$-dimensional Cartesian space.

$$
M(q) \ddot{x}_{r}+C(q, \dot{q}) \dot{x}_{r}+G(q)=F_{c}+F_{e x t}
$$

where $q \in \mathbb{R}^{n}$ denotes the joint configuration and $x_{r} \in \mathbb{R}^{m}$ the robot pose. Moreover, $M \in \mathbb{R}^{m \times m}$ is the mass matrix, $C \in$ $\mathbb{R}^{m \times m}$ represents the centrifugal forces, and $G \in \mathbb{R}^{m}$ denotes the gravitation forces. On the right-hand side, $F_{c}$ and $F_{\text {ext }} \in$

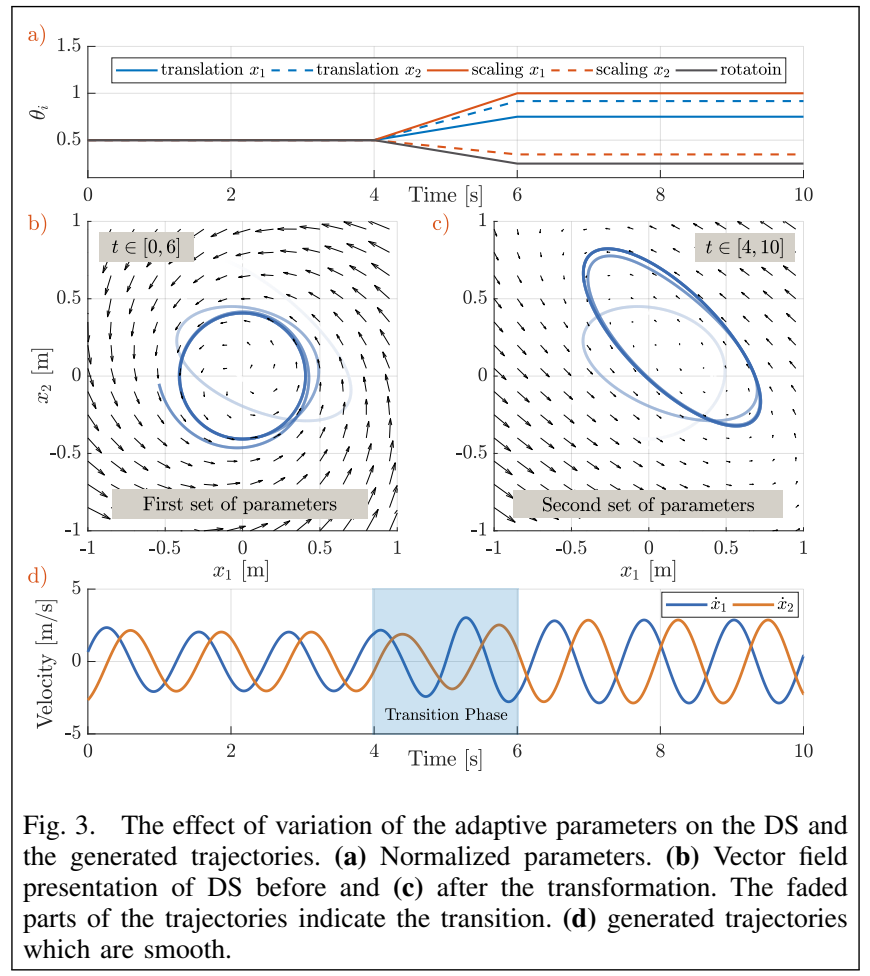

$\mathbb{R}^{m}$ represent the control and external wrenches respectively. We use the following impedance controller proposed by [29] which provides stable and passive physical interaction.

$$
F_{c}=-D\left(\dot{x}_{r}-\dot{x}_{d}\right)+G(q)
$$

where $D \in \mathbb{R}^{m \times m}$ is diagonal with positive entries. $\dot{x}_{d}$ is the desired velocity generated by dynamical systems.

\section{B. Motion Generation}

Consider a desired behavior encoded using statedependent DS as $\dot{x}_{d}=\hat{f}\left(x_{r}\right)$ where $\hat{f}\left(x_{r}\right): \mathbb{R}^{m} \rightarrow \mathbb{R}^{m}$ is a globally stable DS at an attractor or a limit cycle under a continuously differentiable Lyapunov function. To reshape the generated motion by the DS, we consider a diffeomorphism $T(\Theta): \mathbb{R}^{m} \rightarrow \mathbb{R}^{m}$ with $p$ free parameters $\left(\Theta=\left[\theta_{1} \cdots \theta_{p}\right]\right.$ with $\left.\theta_{i} \in \mathbb{R}\right)$. The transformed DS can be formulated as

$$
\dot{x}_{d}=f\left(x_{r} ; \Theta\right)=T(\Theta)^{-1} * \hat{f}\left(T(\Theta) * x_{r}\right)
$$

Moreover, we assume that the DS stability is persevered under the transformation for the region of interest in $\Theta$. In this work, we consider a simple transformation which is a combination of translation, scaling, and rotation. In the following, we provide more details on two types of DS that we use for polishing and pick-and-place tasks.

1) Polishing task: To generate polishing patterns on a surface $\left(x=\left(x_{1}, x_{2}\right)^{T}\right)$,we use a DS described in the polar coordinates $(r$ and $\theta)$ as $\dot{r}=-\alpha\left(r-r_{0}\right)$ and $\dot{\phi}=\omega$ where $r^{2}=x_{1}^{2}+x_{2}^{2}$ and $\phi=\operatorname{atan} 2\left(x_{2}, x_{1}\right) . \omega \in \mathbb{R}^{+}$is the desired angular velocity, $\alpha \in \mathbb{R}^{+}$is the desired radial velocity and $r_{0} \in \mathbb{R}^{+}$is the desired radius of rotation. Fig. 3 shows the adaptation of such a dynamical system. We illustrate the transformation of a simple circular motion to a complex 
cyclic motion through smooth modification of the parameters as described previously. We can observe that the generated trajectories are smooth.

2) Pick-and-place: In order to generate pick-and-place motion for the end-effector position, we use linear dynamics described by $\dot{x}_{d}=-K_{p}\left(x_{r}-\bar{x}\right)$ where $K_{p} \in \mathbb{R}^{3}$ is diagonal with positive entries, and $\bar{x}$ is the target location which is an adaptive parameter. We use three instances of the same dynamics to generate 1) approaching the picking location 2) going through a via-point 3) approaching the placing location. We switch to the next dynamics when we are close enough to the attractor (i.e., $\left\|x_{r}-\bar{x}\right\|<\delta_{p}$ ). Moreover, we go through the via point between each pick and place.

\section{Adaptation Mechanism}

We construct our adaptive law based on a minimization of the tracking error described as $J\left(\Theta ; K, \Delta t_{s}\right)=\frac{1}{2} e^{T} e$ where where

$$
e(t)=\frac{1}{K} \sum_{k=0}^{K-1}\left[f\left(x_{r}\left(t-k \Delta t_{s}\right) ; \Theta\right)-\dot{x}_{r}\left(t-k \Delta t_{s}\right)\right]
$$

is the error between the desired velocity (generated by DS) and the real velocity (influenced by human interaction) over $K$ points spaced with $\Delta t_{s}$ in the past. For example, having $K=1$ brings us back to the instantaneous error. Intuitively, by reducing this error, we adapt the DS to generate the same movements as demonstrated by the human. We obtain this by following the gradient of the cost-function as follows.

$$
\frac{\partial J}{\partial \theta_{i}}=\frac{1}{K} e^{T} \sum_{k=0}^{K-1} \frac{\partial f\left(x_{r}\left(t-k \Delta t_{s}\right) ; \Theta\right)}{\partial \theta_{i}}
$$

To have the sensitivity of the DS to the parameters, we use the following simple approximation.

$$
\frac{\partial f\left(x_{r} ; \Theta\right)}{\partial \theta_{i}}=\frac{f\left(x_{r} ; \Theta_{\neq i}, \theta_{i}+h\right)-f\left(x_{r} ; \Theta_{\neq i}, \theta_{i}-h\right)}{2 h}
$$

where $h$ is the step size of the gradient, and $\Theta_{\neq i}$ denotes all other parameters (except $\theta_{i}$ ) that are kept fixed.

Having the gradient, we update the $i^{\text {th }}$ parameter as follows.

$$
\theta_{i}(t)=\theta_{i}(t-\Delta t)-\varepsilon \frac{\partial J}{\partial \theta_{i}} \Delta t
$$

where $\varepsilon$ and $\Delta t \in \mathbb{R}^{+}$are the adaptation and update rate respectively.

1) Convergence behavior: The convergence behavior of our method can be investigated by assuming the following form for the real velocity.

$$
\dot{x}_{r}=(1-\alpha) \dot{x}_{d}+\alpha f\left(x_{r} ; \Theta^{\star}\right)+\eta(t)
$$

where $\alpha \in[0,1]$ is a rate at which the human takes the robot away from its desired behavior $\left(\dot{x}_{d}=f\left(x_{r} ; \Theta\right)\right)$ and demonstrate his/her intention which we assume follows the same dynamical system but with a different set of parameters $\left(\Theta^{\star}\right) . \eta(t)$ accounts for un-modeled behaviors (caused by the controller and the human). If we linearize w.r.t. $\Theta^{\star}$, we obtain:

$$
f(x ; \Theta)=f\left(x ; \Theta^{\star}\right)+\frac{\partial f(x ; \Theta)}{\partial \Theta}\left(\Theta-\Theta^{\star}\right)+H(x ; \Theta)
$$

where $H($.$) denotes the higher-order terms. Plugging Eq. 8$ and 9 into Eq. 4, simplifies the error function to:

$$
e(t)=S\left(\Theta-\Theta^{\star}\right)+d(t)
$$

where

$$
\left\{\begin{array}{l}
S=\frac{\alpha}{K} \sum_{k=0}^{K-1} \partial f\left(x_{r}\left(t-k \Delta t_{s}\right) ; \Theta\right) / \partial \Theta \\
d(t)=\frac{1}{K} \sum_{k=0}^{K-1} \alpha H\left(x_{r}\left(t-k \Delta t_{s}\right) ; \Theta\right)-\eta\left(t-k \Delta t_{s}\right)
\end{array}\right.
$$

Given the assumption that the disturbance term $d(t)$ is negligible (more precisely, $\partial d / \partial \Theta \simeq 0$ and $S^{T} d \simeq 0$ ) the dynamics of the adaptation (Eq. 7) can be approximated by

$$
\dot{\Theta}=-\varepsilon S^{T} S\left(\Theta-\Theta^{\star}\right)
$$

where $S^{T} S \in R^{p \times p}$ is a positive semidefinite matrix. Given the fact that the number of parameters is higher than the dimension of the error signal $(p>m)$, the rank of $S^{T} S$ is limited to $m$. However, the convergence to $\Theta^{\star}$ can take place if the condition for Persistence Excitation (PE) ([30]) holds as follows.

$$
\exists \delta \quad \forall t \exists T>0 \quad \text { s.t. } \quad \int_{t}^{t+T} S(\tau)^{T} S(\tau) d \tau>\delta I_{m}
$$

This guarantees the convergence of the parameters in average over time. This means that the average of $S^{T} S$ over time is strictly positive definite providing a sufficient condition for stability in Eq.12. To provide better conditions for $P E$ we aim to use a higher number of data-point (i.e., $K$ and $\Delta t_{s}$ ) over a period of time that captures the behavior of both DS and human demonstration. Moreover, the human can improve the convergence by providing demonstration that results in lower $\|d(t)\|$; i.e., demonstrations that can be expressed by the DS (with lower $\|\eta(t)\|$ in Eq.8) and desirable parameters $\left(\Theta^{\star}\right)$ that are close to the current ones (which results in smaller $\|H(x ; \Theta)\|$ in Eq.9).

\section{RESULTS}

The adaptation mechanism is implemented and tested on the Kuka LWR 4+, 7-DOF robotic arm, for two previously described tasks: polishing a surface and pick-and-place. References to the demonstrations of the two tasks can be found in Section V. To activate the adaptation upon human interactions, we used a simple threshold on external force (i.e., $\left\|F_{\text {ext }}\right\|>10 N$ ). Therefore, we avoid to adapt to small tracking errors caused by other uncertainties and mismatched dynamics. In both experiments, the velocities are limited to $0.2 \mathrm{~m} / \mathrm{s}$.

\section{A. Polishing task}

Fig. 4 shows the results for the polishing experiment where the human interacts with robot in three separate intervals (i.e., shaded ares). It can be seen by an increase in the external forces and consequently the tracking error in Fig. 4.a. The tracking error can be investigated further by inspecting the desired and real velocities depicted in Fig. 4.b. Due to the robot compliance, the human is able to demonstrate his/her intention by influencing the real velocity. Fig. 4.c shows the 


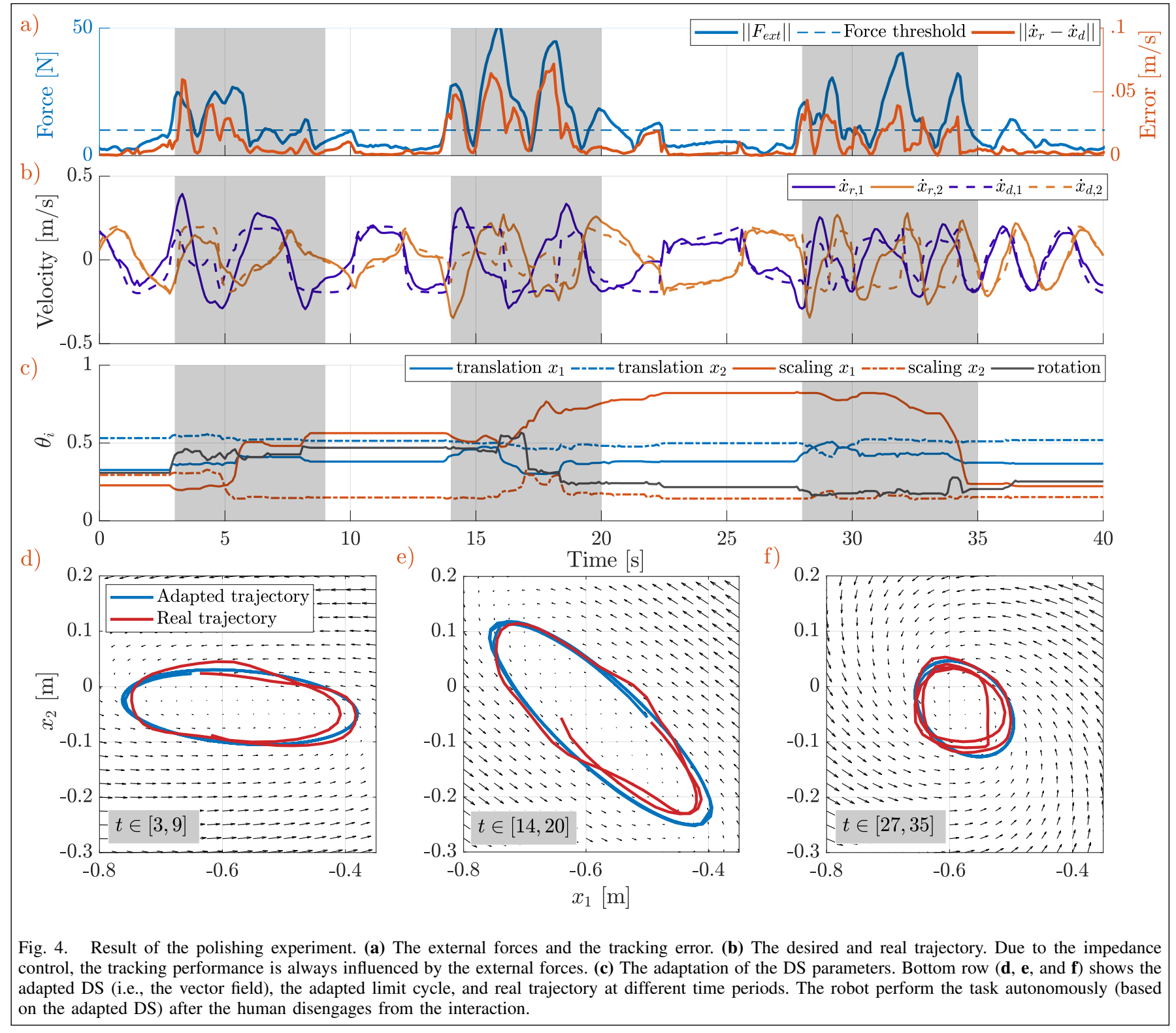

adaptation of the parameters which consist of the translation and scaling along both axes and the rotation. It can be seen that upon interacting with the robot, the parameters are adapting in order to reduce the error. The bottom row of Fig. 4 shows the state of the DS in the three corresponding intervals where the human interacts with the robot. These plots demonstrate the ability of our method to fit the DS (i.e., the vector field) to the demonstrated motions; i.e., to capture the intention of the human use. Moreover, these plots show that the parameterized DS is capable of generating different polishing patterns (i.e., limit cycles). In this experiment, the following hyper-parameters are used: $\varepsilon=0.1, h=0.01$, $\Delta t_{s}=5 \mathrm{~s}, K=10$, and $\Delta t=0.05 \mathrm{~s}$.

\section{B. Pick-and-place}

Fig. 5 shows the results of our implementation for repetitive pick-and-place where the robot alternates between pick and place motion while going through a via-point. We use our algorithm to adapt the target locations of pick and place. Fig. 5.a. show the external forces and resulting tracking errors induced by the human user. The desired velocity generated by DS and the real velocity influenced partially by the user are illustrated in Fig. 5.b. Fig. 5.c shows the adaptation of the normalized parameters: the pick and place target locations. It can be seen that only the parameters of the active DS (either pick or place) are being adapted. Fig. 5.d illustrate the human-robot interaction between $2 s$ and $12 s$. After performing pick, via-point, place, via-point, the robot reaches for the last picking target. However, upon human interaction (the human pulls the robot to a new intended location), the target location for picking adapts accordingly. Fig. 5.e-f show similar instances of such interactions where the target locations are adapted. In this experiment, we use the following hyper-parameters: $\varepsilon=0.001, h=0.01, K=1$ ( $\Delta t_{s}$ not applicable), $\Delta t=0.05 s$, and $K_{p}=2$. Moreover, 


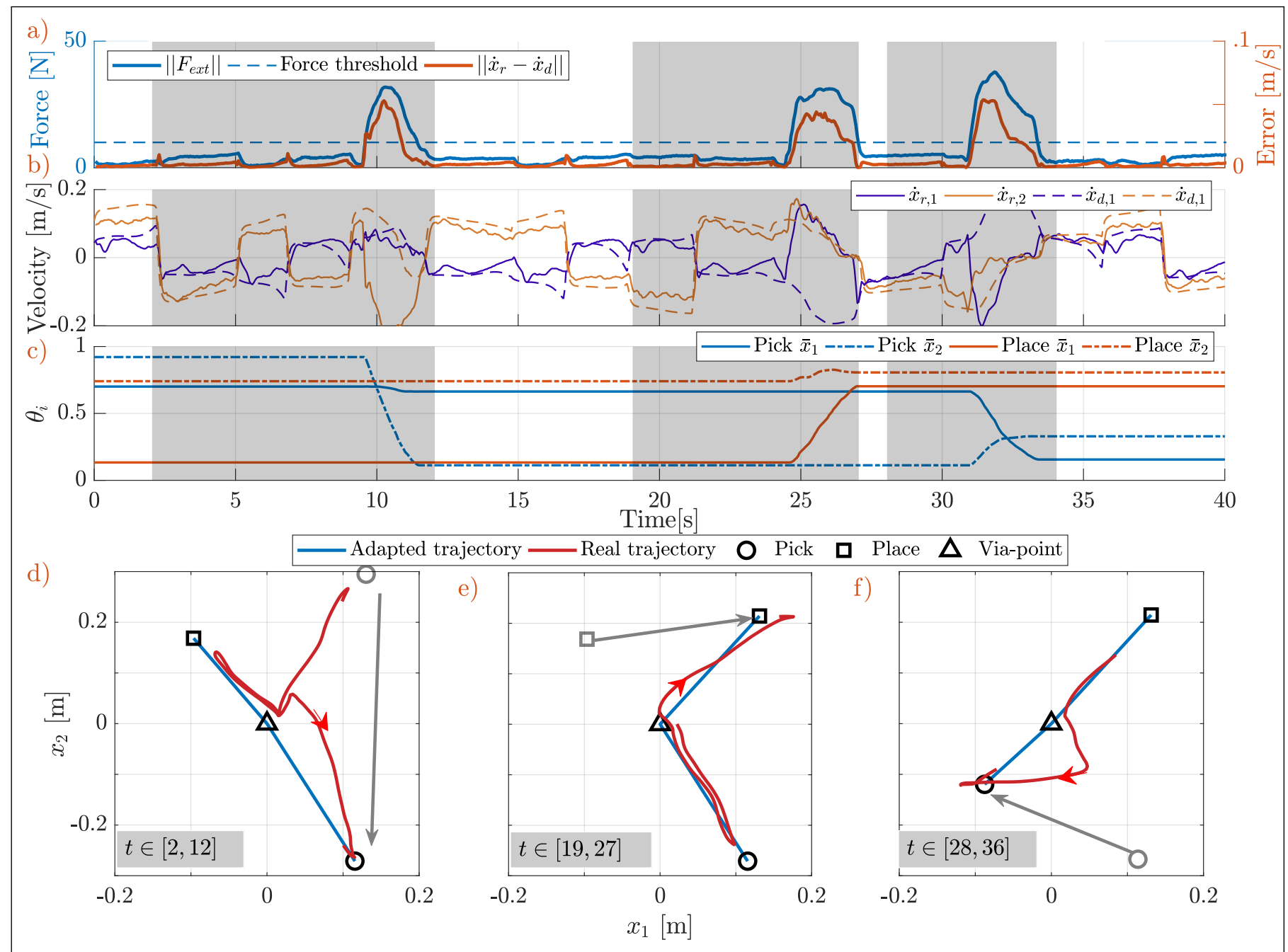

Fig. 5. Result of the pick-and-place experiment. (a) The external forces and the tracking error. (b) The desired and real trajectory. (c) The adaptation of the DS parameters. Bottom row (d, e, and f) shows the adapted target locations, and real trajectory at different time periods.

in this implementation, the post-condition for switching between DSs (e.g., from pick to via-point) is (1) to reach the target $\left(\| x_{r}-x \overline{\|} \mid<0.05 m\right)$ and (2) the absence of human interaction $\left(|| F_{h} \|<10 N\right)$. This post-condition allows the human user to adapt the current DS and not switch to the next DS even if it reaches its target.

\section{DISCUSSION AND CONCLUSION}

In this work, we derived our adaptive mechanism based on tracking error where the performance of the adaptation can be tuned using its set of hyper-parameters. Here, we provide a short discussion on the effect of these hyperparameters. The speed of convergence is primarily controlled by the adaptation rate $(\varepsilon)$ which represents a trade-off between slow convergence and fluctuations in the estimated parameters. The approximation of the gradient is tuned by $h$. This parameter needs to be small enough to have a precise estimation of the gradient. However, dealing with a noisy cost-function (due to noisy $\dot{x}_{r}$ ), it needs to be big enough to avoid over-fitting (i.e., to the noise). Finally $\Delta t_{s}$ and $K$ can be tuned properly to provide a rich signal for adaptation (Eq. 13). $\Delta t_{s}$ needs to be large enough to capture the characteristics of both DS and human intention. For example, in the polishing task, we use a $\Delta t_{s}$ that includes enough samples from a complete cycle of the motion. Further increment of $\Delta t_{s}$ includes information from the past that are no longer relevant. $K$ controls the number of samples from the time-window created by $\Delta t_{s} . K$ needs to be tuned properly to have a robust down-sampling while reducing the computational cost. For example, we found $K=1$ to be sufficient in the pick-and-place experiment. Intuitively, the condition for persistent excitation is easier to achieve: input dimension being 2 (i.e., error along $x_{1}$ and $x_{2}$ ) and parameter dimension being 2 (i.e., $\bar{x}_{1}$ and $\bar{x}_{2}$ since the adaptations of pick and place are mutually exclusive) compared to polishing task where the input dimension is 2, however, there are 5 parameters to adapt. This fact is reflected in the convergence speed of the parameters; compare Fig. 4.c with Fig. 5 where the latter has faster convergence. Finally, in order to distinguish the human interaction from other undesirable forces (disturbances, frictional forces, etc.), the 
force-threshold needs to be chosen carefully. A low value results in undesirable adaptation to disturbances, while a high value requires higher human effort to trigger the adaptation. A more sophisticated and robust method for the detection of human interaction is on our list for future work.

Overall, the proposed adaptive mechanism enables the robot to adapt its motion according to the human interactions. DS-based Impedance controller, along with a transformation that preserves the stability of the DS, guarantees the overall stability of the control loop. Moreover, the convergence of the parameters (to the intended ones) is guaranteed if the human demonstration satisfies the persistent excitation condition. Our implementation on the robotic arm for different tasks (i.e., polishing a surface and pick-and-place) proves the efficacy of our method in capturing the human intention. Our experimental results are in line with our analytical analysis in terms of convergence behavior. In conclusion, parameterized dynamical systems (as adaptive motion generators) along with impedance control (providing compliant interaction) proves to be effective to provide seamless and intuitive physical human-robot interaction. In future, we focus on reducing human effort and improving tracking performance since in the current state, the impedance gain presents a trade-off between compliance and tracking precision.

\section{SUPPLEMENTARY MATERIAL}

Demonstrations of our method can be viewed in the video attachments or at https://youtu.be/qICOAtVMNgE and https://youtu.be/TGwNkSEMm0M

\section{ACKNOWLEDGMENT}

We thank the European Community Horizon 2020 Research and Innovation programme ICT-23-2014 643950SecondHands, and Guillaume deChambrier and Klas Kronander for their implementations of the Kuka LWR controller.

\section{REFERENCES}

[1] A. Cherubini, R. Passama, A. Crosnier, A. Lasnier, and P. Fraisse, "Collaborative manufacturing with physical human-robot interaction," Robotics and Computer-Integrated Manufacturing, vol. 40, pp. 1-13, 2016.

[2] N. Hogan, "Impedance control: An approach to manipulation: Part iiimplementation," Journal of dynamic systems, measurement, and control, vol. 107, no. 1, pp. 8-16, 1985.

[3] F. J. Abu-Dakka, B. Nemec, J. A. Jørgensen, T. R. Savarimuthu, N. Krüger, and A. Ude, "Adaptation of manipulation skills in physical contact with the environment to reference force profiles," Autonomous Robots, vol. 39, no. 2, pp. 199-217, 2015.

[4] G. Ganesh, A. Albu-Schäffer, M. Haruno, M. Kawato, and E. Burdet, "Biomimetic motor behavior for simultaneous adaptation of force, impedance and trajectory in interaction tasks," in ICRA. IEEE, 2010, pp. 2705-2711.

[5] B. Corteville, E. Aertbeliën, H. Bruyninckx, J. De Schutter, and H. Van Brussel, "Human-inspired robot assistant for fast point-to-point movements," in Robotics and Automation, 2007 IEEE International Conference on. IEEE, 2007, pp. 3639-3644.

[6] F. Abi-Farraj, T. Osa, N. P. J. Peters, G. Neumann, and P. R. Giordano, "A learning-based shared control architecture for interactive task execution," in ICRA. IEEE, 2017, pp. 329-335.

[7] G. Maeda, M. Ewerton, T. Osa, B. Busch, and J. Peters, "Active incremental learning of robot movement primitives," in Conference on Robot Learning (CORL), 2017.
[8] D. Lee and C. Ott, "Incremental motion primitive learning by physical coaching using impedance control," in IROS, 2010, pp. 4133-4140.

[9] A. Ghadirzadeh, J. Bütepage, A. Maki, D. Kragic, and M. Björkman, "A sensorimotor reinforcement learning framework for physical human-robot interaction," in IROS. IEEE, 2016, pp. 2682-2688.

[10] M. Hazara and V. Kyrki, "Reinforcement learning for improving imitated in-contact skills," in Humanoids 2016, 2016, pp. 194-201.

[11] S. Jlassi, S. Tliba, and Y. Chitour, "An online trajectory generatorbased impedance control for co-manipulation tasks," in Haptics Symposium (HAPTICS), 2014 IEEE, 2014, pp. 391-396.

[12] E. L. Sauser, B. D. Argall, G. Metta, and A. G. Billard, "Iterative learning of grasp adaptation through human corrections," Robotics and Autonomous Systems, vol. 60, no. 1, pp. 55-71, 2012.

[13] S. Cho and S. Jo, "Incremental online learning of robot behaviors from selected multiple kinesthetic teaching trials," IEEE Transactions on Systems, Man, and Cybernetics, vol. 43, no. 3, pp. 730-740, 2013.

[14] M. Ewerton, G. Maeda, G. Kollegger, J. Wiemeyer, and J. Peters, "Incremental imitation learning of context-dependent motor skills," in Humanoid Robots (Humanoids), 2016 IEEE-RAS 16th International Conference on. IEEE, 2016, pp. 351-358.

[15] M. Tykal, A. Montebelli, and V. Kyrki, "Incrementally assisted kinesthetic teaching for programming by demonstration," in The Eleventh ACM/IEEE International Conference on Human Robot Interaction. IEEE Press, 2016, pp. 205-212.

[16] D. Lee and C. Ott, "Incremental kinesthetic teaching of motion primitives using the motion refinement tube," Autonomous Robots, vol. 31, no. 2-3, pp. 115-131, 2011.

[17] A. K. Tanwani and S. Calinon, "A generative model for intention recognition and manipulation assistance in teleoperation," in IROS. IEEE, 2017, pp. 43-50.

[18] E. Noohi and M. Žefran, "Estimating human intention during a human-robot cooperative task based on the internal force internal force model," in Trends in Control and Decision-Making for Human-Robot Collaboration Systems. Springer, 2017, pp. 83-109.

[19] A. Bussy, P. Gergondet, A. Kheddar, F. Keith, and A. Crosnier, "Proactive behavior of a humanoid robot in a haptic transportation task with a human partner," in RO-MAN. IEEE, 2012, pp. 962-967.

[20] M. Khoramshahi and M. Billard, "A dynamical system approach to task-adaptation in physical human-robot interaction," in under review in Autonomous Robots. Springer, 20178.

[21] A. M. Kabir, K. N. Kaipa, J. Marvel, and S. K. Gupta, "Automated planning for robotic cleaning using multiple setups and oscillatory tool motions," IEEE Transactions on Automation Science and Engineering, vol. 14, no. 3, pp. 1364-1377, 2017.

[22] Y. Oba, Y. Yamada, K. Igarashi, S. Katsura, and Y. Kakinuma, "Replication of skilled polishing technique with serial-parallel mechanism polishing machine," Precision Engineering, vol. 45, pp. 292-300, 2016.

[23] C. Schindlbeck and S. Haddadin, "Unified passivity-based cartesian force/impedance control for rigid and flexible joint robots via taskenergy tanks," in ICRA. IEEE, 2015, pp. 440-447.

[24] C. P. Quintero, R. Tatsambon, M. Gridseth, and M. Jägersand, "Visual pointing gestures for bi-directional human robot interaction in a pickand-place task," in International Symposium on Robot and Human Interactive Communication (RO-MAN), 2015, pp. 349-354.

[25] R. Schulz, "Collaborative robots learning spatial language for picking and placing objects on a table," in Proceedings of the 5th International Conference on Human Agent Interaction. ACM, 2017, pp. 329-333.

[26] L. Peternel, N. Tsagarakis, D. Caldwell, and A. Ajoudani, "Robot adaptation to human physical fatigue in human-robot comanipulation," Autonomous Robots, pp. 1-11, 2017.

[27] A. Gams, T. Petric, B. Nemec, and A. Ude, "Learning and adaptation of periodic motion primitives based on force feedback and human coaching interaction," in Humanoid Robots (Humanoids), 2014 14th IEEE-RAS International Conference on. IEEE, 2014, pp. 166-171.

[28] S. M. Khansari-Zadeh and A. Billard, "Learning stable nonlinear dynamical systems with gaussian mixture models," IEEE Transactions on Robotics, vol. 27, no. 5, pp. 943-957, 2011.

[29] K. Kronander and A. Billard, "Passive interaction control with dynamical systems," IEEE Robotics and Automation Letters, vol. 1, no. 1, pp. 106-113, 2016.

[30] K. J. Åström and B. Wittenmark, Adaptive control. Courier Corporation, 2013. 